\title{
Fibrinoid Leukodystrophy (Alexander's Disease-Like Disorder) in a Young Adult French Bulldog
}

\author{
Tsuyoshi ITO ${ }^{1)}$, Kazuyuki UCHIDA ${ }^{1) *}$, Masatoshi NAKAMURA ${ }^{2)}$, Ko NAKASHIMA ${ }^{2)}$, Kazuhiko SUZUKI ${ }^{1)}$ and \\ Hiroyuki NAKAYAMA ${ }^{1)}$ \\ Departments of ${ }^{1)}$ Veterinary Pathology and ${ }^{2)}$ Internal Medicine, Graduate School of Agricultural and Life Sciences, The University of \\ Tokyo, 1-1-1 Yayoi, Bunkyo-Ku, Tokyo 113-8657, Japan
}

(Received 1 March 2010/Accepted 22 May 2010/Published online in J-STAGE 4 June 2010)

ABSTRACT. The paper describes clinical and pathological features of Alexander's disease (AD)-like disorder in a 1 year and 8 months old French bulldog. Clinically, the dog exhibited megaesophagus, emaciation and weakness without any specific neurological symptoms. The dog died of aspiration pneumonia. On the gross observation of formalin-fixed brain, discolored foci were observed in the white matter of the cerebellum and brain stem. Histologically, numerous Rothenthal fibers and hypertrophic astrocytes were distributed especially in the perivascular, subependymal and subpial area of both the cerebrum and cerebellum. The Rosenthal fibers were intensely immunopositive for GFAP and ubiquitin. Demyelination of the white matter was occasionally found in the brain stem. The present case is likely to be categorized in the adult form of $\mathrm{AD}$, though previous $\mathrm{AD}$-like cases in dogs were in the juvenile form. KEY WORDS: adult-onset form, Alexander's disease, canine, Rothenthal fibers.

J. Vet. Med. Sci. 72(10): 1387-1390, 2010

Human Alexander's disease (AD) is an infantile leukodystrophy which was first described in 1949 and characterized by macrocephaly, psychomotor regression, spasticity, ataxia and seizures leading to death within a few years [6, 11]. Characteristic gross changes are grayish discoloration of the white matter, especially in the prosencephalon and ventricular dilatation. Microscopically, widespread myelin pallor along with characteristic Rosenthal fibers (RFs) are observed throughout the central nervous system (CNS) [14]. Transgenic mice overexpressing glial fibrillary acidic protein (GFAP) develop RFs [8], and gene mutations in GFAP seem to be most relevant to the cause of AD. The disease is classified based on the age of onset; i) the infantile form with megalencephaly and developmental delay at birth to 2 years old, ii) the juvenile form with seizures, bulbar signs and/or mental deteriolation at 2 to 12 years old, and iii) the adult-onset form with slowly progressive bulbar dysfunction and various neurological signs in the second to seventh decade of life [13].

A few cases clinically and morphologically identical to $\mathrm{AD}$ in humans have been reported in dogs and a sheep [3]. All of the dog cases $[1,2,7,12,18]$ developed the AD-like disorders by 6-month-old, thus they were considered to resemble the juvenile form of $\mathrm{AD}$ (Table 1). The present paper describes a caine case of adult-onset AD.

A 1 year and 8 months old, spayed female French bulldog showed vomiting 2 month before death, and developed gradual weight loss. By barium contrast examination, megaesophagus was observed, suspecting to develop aspiration pneumonia. Despite of fluid food supply, vomiting did

\footnotetext{
* Correspondence to: Uchida, K., Department of Veterinary Pathology, Graduate School of Agricultural and Life Sciences, The University of Tokyo, 1-1-1 Yayoi, Bunkyo-Ku, Tokyo 113-8657, Japan.

e-mail: auchidak@mail.ecc.u-tokyo.ac.jp
}

not improve. Anti-acetylcholine receptor antibody titer in the serum was $0.12 \mathrm{nmol} / l$ (normal range: $0-0.6$ ). Neurological examinations revealed no specific abnormality. The dog became emaciated and died.

At necropsy, the esophagus was markedly enlarged with attenuation of the wall along its entire length. The lungs were entirely reddish brown in color, and foamy contents accumulated in their bronchial tubes. There were scattered foci of discoloration on the cut surface of the formalin-fixed cerebellum and brain stem (Fig. 1). The foci in the brain stem appeared translucent.

The tissue samples were fixed in $10 \%$ neutral buffered formalin solution for 4 days, processed routinely, embedded in paraffin, sectioned at $4 \mu \mathrm{m}$-thick, and stained with hematoxylin and eosin (HE). The brain sections were also stained with luxol fast blue-HE (LFB-HE). Immunohistochemical stainings were applied on the representative sections. The primary antibodies used were rabbit anti-GFAP (1:400, Dako, Glostrup, Denmark), anti-ubiquitin (1:200, Dako) and anti-CD20 (1:400, Thermo Scientific, Fremont, CA, U.S.A.). Following a treatment with $8 \%$ skim milk at $37^{\circ} \mathrm{C}$ for $40 \mathrm{~min}$, sections were incubated with the primary antibodies at $4{ }^{\circ} \mathrm{C}$ overnight, and followed by secondary antibody reactions at $37^{\circ} \mathrm{C}$ for $40 \mathrm{~min}$ using EnVision AntiRabbit Conjugation system (Dako, Carpinteria, CA, U.S.A.). Finally, the positive reactions were visualized with $0.05 \% 3,3$ '-diaminobenzidine (DAB) and $0.03 \%$ hydrogen peroxide in Tris- $\mathrm{HCl}$ buffer, and counterstained with hematoxylin.

Histopathological lesions were distributed throughout the white matter of the brain (Fig. 2), especially in the cerebellum and brain stem. Numerous eosinophilic RFs were shown mostly in the perivascular, subependymal and subpial areas along with frequent distribution of abnormally 
Table 1. Characteristics of animals with leukodystrophy resembling Alexander's disease in the literature

\begin{tabular}{|c|c|c|c|c|c|}
\hline Specie & Breeds & Age onset & Age dead & Clinical signs & Reference No. \\
\hline \multirow{7}{*}{ Dog } & Labrador retriever & $6 \mathrm{~m}^{*}$ & 11 m* (euthanatized) & \multirow[t]{2}{*}{ Ataxia, pelvic limb paresis } & \multirow[t]{2}{*}{7} \\
\hline & & $6 \mathrm{~m}^{*}$ & $16 \mathrm{~m} *$ (euthanatized) & & \\
\hline & Scottish terrier & $6 \mathrm{~m}^{*}$ & 9 m* (euthanatized) & Ataxia, unable to stand & 2 \\
\hline & Miniature poodle & $3 \mathrm{~m}^{*}$ & $6 \mathrm{~m} *$ (euthanatized) & Ataxia, posterior weakness & 12 \\
\hline & Bernese mountain dog & $2 \mathrm{~m} 1 \mathrm{w}^{*}$ & $3 \mathrm{~m} 1 \mathrm{w}^{*}$ (euthanatized) & Posterior weakness, tetraplegia & 18 \\
\hline & Bernese mountain dog & $3 \mathrm{~m} 1 \mathrm{w} *^{*}$ & $4 \mathrm{~m} *$ (euthanatized) & Tetraparesis, generalized tremors & 1 \\
\hline & French bulldog & $18 \mathrm{~m} *$ & $20 \mathrm{~m} *$ & Ataxia, megaesophagus & Present case \\
\hline Sheep & White Alpine & $4 y^{*}$ & $4 y^{*}$ (at the 3rd day after admission) & Astasia, somnolence, hyperexcitability & ty 3 \\
\hline
\end{tabular}

*; y: years, m: months, and w: weeks.

enlarged astrocytes (Fig. 3). Immunohistochemically, the RFs were intensely positive for GFAP and ubiquitin (Fig. 4a and 4 b). Some enlarged astrocytes contained a large amount of GFAP- and ubiquitin-positive materials in their cytoplasm (Fig. 4a and 4b, insets), indicating the presence of ubiquitinated GFAP within astrocytes. In the cerebral cortex, hypertrophic astrocytes, which were positive for GFAP, diffusely proliferated. Demyelinating foci were occasionally disclosed in the brain stem on the LFB-HE stained sections (Fig. 5). In the cerebellum and brain stem, perivascular infiltration of lymphocytes and hemorrhagic foci were also occasionally observed (Fig. 6). Most of the perivascular lymphocytes were immunopositive for CD20 suggesting B cells (Fig. 6).

Human AD is included in the category of leukodystrophy. The main microscopic features of human cases are GFAPand ubiquitin-positive RFs and hypertrophic astrocytes. Human AD is classified according to the age onset; as infantile-, juvenile- and adult-onset forms. Each form of the disease is also characterized by the particular histological features. In the infantile form which is most severely affected, the entire CNS rapidly degenerates causing intracranial hypertension, epileptic seizure, motor neuron impairment, and cognitive decline. Extensive loss of white matter is observed. The adult form is characterized by predominant rhombencephalic degeneration without epilepsy and cognitive impairment. White matter loss is little, if any. The juvenile form is intermediate between the two forms [9]. In dogs, AD-like disorders have been reported in the Labrador retriever, Scottish terrier, miniature poodle and Bernese mountain dog (Table 1). The dogs developed symptoms such as ataxia, paresis and tremor from juvenile ages. Histologically, white matter demyelination was observed together with a large amount of RFs and astrogliosis. These findings were similar to those of the juvenile form of human $\mathrm{AD}$. In the present case, the first symptom, megaesophagus, was observed at 1 year and 6 months of age, which is the young adult category in dogs. In the brain, the lesion is observed mainly in the brain stem and white matter demyelination was minimal. These findings might suggest that the present case resembles the adult form of human $\mathrm{AD}$ rather than juvenile form. In nonhuman animals, the adult-onset of AD-like disorder was only reported in a 4year-old ewe [3].

Most of the infantile and juvenile forms of human AD are sporadic, while familial distribution is often suspected for the adult form [4]. In dog cases, two Labrador retriever littermates indicated similar histological changes resembling AD [7]. Additionally, three of four littermates of Scottish terrier showed AD-like neurological symptoms, although they were not pathologically examined [2]. Canine AD-like disorders may also be heritable. Further molecular biological studies for the GFAP gene will be necessary.

The present case developed marked megaesophagus. If the brain stem motor nuclei are affected, multiple symptoms such as dysphonia, dysphagia, megaesophagus, and external ophthalmoplegia may occur [10]. However, the present case showed only megaesophagus, not other multiple symptoms. Thus, we suppose that megaesophagus might be an unrelated congenital disorder.

In the present case, perivascular CD20-positive B cell infiltration was observed in the brain stem. Such perivascular lymphocytic infiltration has also been reported in human $\mathrm{AD}$ [15], although there have been no information about the change in previous canine AD-like cases $[1,2,7,12,18]$. The evidence of blood-brain-barrier (BBB) disruption has been confirmed by magnetic resonance imaging examinations using contrast-enhancement in human AD patients [9]. Such abnormality of BBB might be associated with lymphocytic infiltration. On the other hand, RFs observed in human AD are immunohistochemically positive for GFAP, ubiquitin, alpha-B-crystalline and heat shock protein (Hsp) 27 [5]. The alpha B-crystalline, a small heat shock protein, is a putative myelin autoantigen in human multiple sclerosis [16] and its phosphorylation at Ser-45 was reported to induce immunological intolerance [17]. In two patients with human $\mathrm{AD}$, the protein was significantly phosphorylated at Ser-59 and Ser-45 [5]. The perivascular B cell aggregation observed in present case may be the immunological response to the phosphorylated alpha B-crystallin. Since immunohistochemical detection of alpha B-crystallin or Hsp 27 was failed due to no cross-reactive antibody to the canine, the exact reason of the lymphoid accumulation in the lesions remains unclear. 

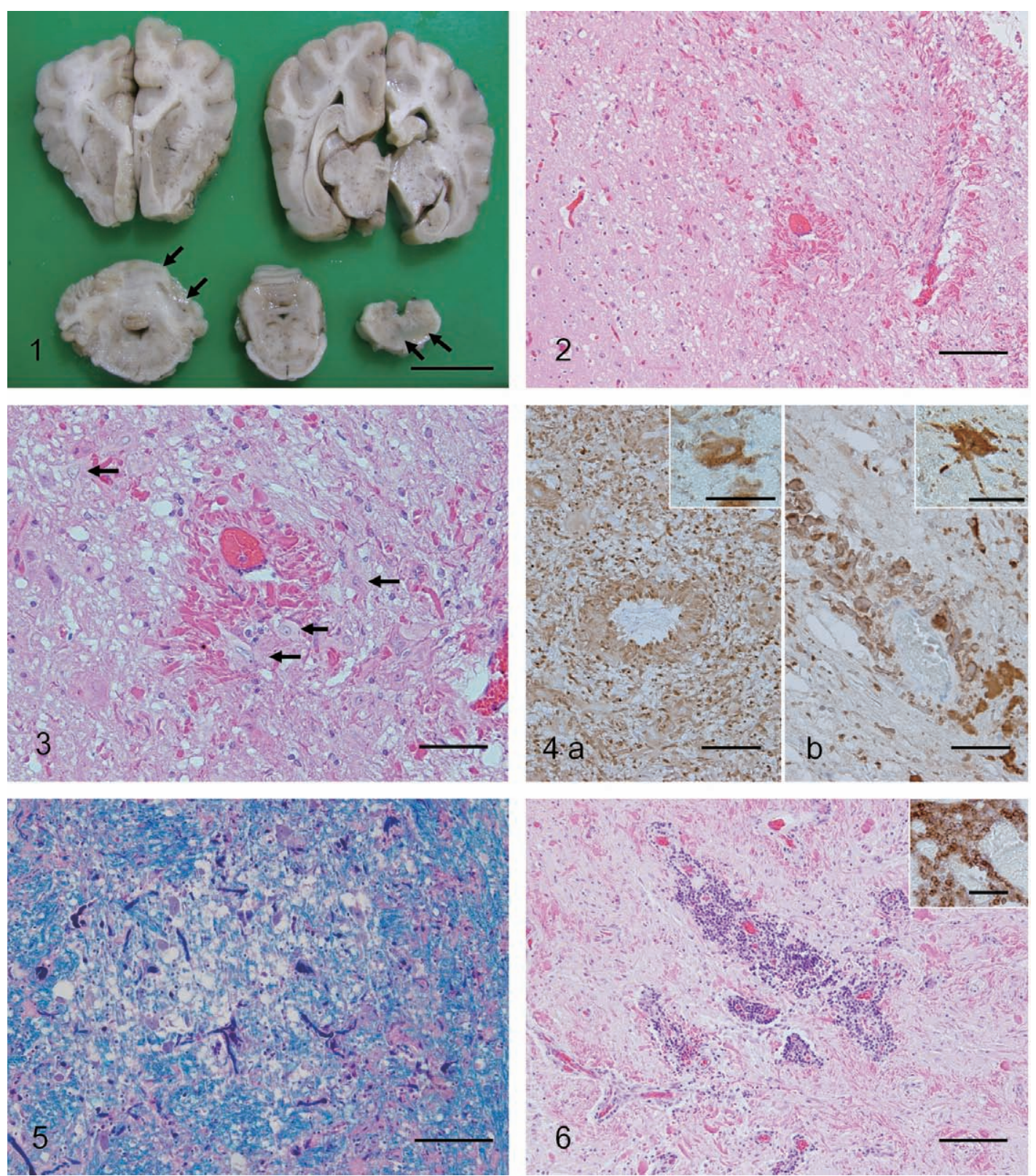

Fig. 1. Brain. Discolored foci are observed in the white matter of cerebellum and medulla oblongata (arrows). The foci in the medulla oblongata are translucent.

Fig. 2. Mesencephalon. Lesions with perivascular RFs are distributed mainly in the white matter. HE. Bar=100 $\mu \mathrm{m}$.

Fig. 3. Mesencephalon. Eosinophilic RFs aggregate mainly around blood vessels. Abnormally hypertrophic astrocytes are also found (arrows). HE. Bar $=50 \mu \mathrm{m}$.

Fig. 4. Cerebrum. a) RFs and abnormally hypertrophic astrocytes are immunopositive for GFAP. EnVison method. Counterstained with Mayer's hematoxylin. Bar $=50 \mu \mathrm{m}$. b) RFs and abnormally hypertrophic astrocytes are also immunopositive for ubiquitin. EnVison method. Counterstained with Mayer's hematoxylin. Bar=50 $\mu \mathrm{m}$. Inset: A hypertrophic astrocyte contains a large amount of GFAP- and ubiquitin-positive materials in the cytoplasm. Bar $=40 \mu \mathrm{m}$.

Fig. 5. Medulla oblongata. Lesions of demyelination. LFB-HE. Bar $=100 \mu \mathrm{m}$.

Fig. 6. Medulla oblongata. In addition to numerous RFs, perivascular lymphocyte infiltration is observed. HE. Bar=100 $\mu \mathrm{m}$. Inset: Perivascular lymphocytes are immunopositive for CD20. EnVison method. Counterstained with Mayer's hematoxylin. Bar $=100 \mu \mathrm{m}$. 


\section{REFERENCES}

1. Alemañ, N., Marcaccini, A., Espino, L., Bermúdez, R., Nieto, J. M. and López-Peña, M. 2006. Rosenthal fiber encephalopathy in a dog resembling Alexander disease in humans. Vet. Pathol. 43: 1025-1028.

2. Cox, N. R., Kwapien, R. P., Sorjonen, D. C. and Braund, K. G. 1986. Myeloencephalopathy resembling Alexander's disease in a Scottish terrier dog. Acta Neuropathol. 71: 163-166.

3. Fankhauser, R., Fatzer, R., Bestetti, G., Deruaz, J. P. and Perentes, E. 1980. Encephalopathy with Rosenthal fibre formation in a sheep. Acta Neuropathol. 50: 57-60.

4. Johnson, A. B. 2002. Alexander disease: a review and the gene. Int. J. Dev. Neurosci. 20: 391-394.

5. Kato, K., Inaguma, Y., Ito, H., Iida, K., Iwamoto, I., Kamei, K., Ochi, N., Ohta, H. and Kishikawa, M. 2001. Ser-59 is the major phosphorylation site in alphaB-crystallin accumulated in the brains of patients with Alexander's disease. J. Neurochem. 76: 730-736.

6. Li, R., Johnson, A. B., Salomons, G., Goldman, J. E., Naidu, S., Quinlan, R., Cree, B., Ruyle, S. Z., Banwell, B., D’Hooghe, M., Siebert, J. R., Rolf, C. M., Cox, H., Reddy, A., GutiérrezSolana, L. G., Collins, A., Weller, R. O., Messing, A., van der Knaap, M. S. and Brenner, M. 2005. Glial fibrillary acidic protein mutations in infantile, juvenile, and adult forms of Alexander disease. Ann. Neurol. 57: 310-326.

7. McGrath, J. T. 1979. Fibrinoid leukodystrophy (Alexander's disease). pp. 147-148. In: Spontaneous Animal Models of Human Disease, Vol. 2 (Andrews, W. J., Ward, B. C. and Altman, H. N. eds.), Academic Press, New York.

8. Messing, A., Head, M. W., Galles, K., Galbreath, E. J., Goldman, J. E. and Brenner, M. 1998. Fatal encephalopathy with astrocyte inclusions in GFAP transgenic mice. Am. J. Pathol.
152: $391-398$

9. Mignot, C., Boespflug-Tanguy, O., Gelot, A., Dautigny, A., Pham-Dinh, D. and Rodriguez, D. 2004. Alexander disease: putative mechanisms of an astrocytic encephalopathy. Cell. Mol. Life. Sci. 61: 369-385.

10. Olby, N. 2004. Motor neuron disease: inherited and acquired. Vet. Clin. North Am. Small Anim. Pract. 34: 1403-1418.

11. Quinlan, R. A., Brenner, M., Goldman, J. E. and Messing, A. 2007. GFAP and its role in Alexander disease. Exp. Cell. Res. 313: 2077-2087.

12. Richardson, J. A., Tang, K. and Burns, D. K. 1991. Myeloencephalopathy with Rosenthal fiber formation in a miniature poodle. Vet. Pathol. 28: 536-538.

13. Sawaishi, Y. 2009. Review of Alexander disease: beyond the classical concept of leukodystrophy. Brain. Dev. 31: 493-498.

14. Summers, A. B., Cummings, J. F. and de Lahunta, A. 1995. Degenerative diseases of the central nervous system. pp. 282283. In: Veterinary Neuropathology, Mosby, St. Louis.

15. Towfighi, J., Young, R., Sassani, J., Ramer, J. and Horoupian, D. S. 1983. Alexander's disease: further light-, and electronmicroscopic observations. Acta Neuropathol. 61: 36-42.

16. van Noort, J. M., van Sechel, A. C., Bajramovic, J. J., el Ouagmiri, M., Polman, C. H., Lassmann, H. and Ravid, R. 1995. The small heat-shock protein alpha B-crystallin as candidate autoantigen in multiple sclerosis. Nature 375: 798-801.

17. van Stipdonk, M. J., Willems, A. A., Amor, S., Persoon-Deen, C., Travers, P. J., Boog, C. J. and van Noort, J. M. 1998. T cells discriminate between differentially phosphorylated forms of alphaB-crystallin, a major central nervous system myelin antigen. Int. Immunol. 10: 943-950.

18. Weissenböck, H., Obermaier, G. and Dahme, E. 1996. Alexander's disease in a Bernese mountain dog. Acta Neuropathol. 91: 200-204. 